\title{
Comparison of Totally Thoracoscopic and Traditional Sternotomy Approaches for Mitral Valve Replacement
}

\author{
He Fan, Qian Xi-Ming, Zhang Wei-Min, Chen Huai-Dong \\ Department of Cardiac Surgery, Sir Run Run Shaw Hospital, School of Medicine, Zhejiang University, Hangzhou, China
}

\section{ABSTRACT}

Background: We aimed to investigate the feasibility and safety of mitral valve replacement using a totally thoracoscopic approach in comparison with traditional median sternotomy.

Methods: Between January 2016 and December 2017, 94 consecutive patients who underwent mitral valve replacement were divided into two groups: A thoracoscopic group (43 cases) and a traditional group (51 cases). For the thoracoscopic group, all patients underwent total thoracoscopic procedures with femoral arterial and venous cannulation to cardiopulmonary bypass, transthoracic aortic cross-clamp, and antegrade cardioplegia. Three intercostal ports in the right chest were used for access in the thoracoscopic group. The operation was performed completely under two-dimensional video. For the traditional group, all operations were done with traditional median sternotomy.

Results: All the operations were successfully performed. The thoracoscopic group had longer aortic cross-clamping and cardiopulmonary bypass times compared with the traditional group $(62.30 \pm 8.17$ minutes versus $44.90 \pm 12.00 \mathrm{~min}-$ utes, $P<.001 ; 92.33 \pm 12.03$ minutes versus $74.22 \pm 14.72$ minutes, $P<.001$ ). The two groups did not show statistically significant differences with respect to operative times (184.26 \pm 32.49 minutes versus $181.47 \pm 23.31$ minutes, $P=.631$ ) In addition, the postoperative mechanical ventilation, ICU stay, and postoperative hospital stay times and postoperative drainage were $10.14 \pm 2.21$ hours and $11.35 \pm 2.58$ hours $(P=.016), 21.40 \pm 3.15$ hours and $29.12 \pm 6.59$ hours $(P<.001)$, $8.70 \pm 2.52$ days and $10.04 \pm 3.11$ days $(P=.023)$, and 325.71 $\pm 97.11 \mathrm{~mL}$ and $396.57 \pm 121.50 \mathrm{~mL}(P<.001)$, respectively. Major postoperative complications occurred in three $(6.98 \%$, $P=.873)$ cases of the thoracoscopic group. Four $(7.84 \%)$ cases of the traditional group had postoperative complications.

Conclusions: Despite the disadvantages such as long cross-clamp and cardiopulmonary bypass times, totally thoracoscopic mitral valve replacement is feasible and safe. More importantly, one of the principal advantages with three intercostal ports over standard sternotomy is avoiding retrosternal

Received February 28, 2019; received in revised form May 20, 2019; accepted May 31, 2019.

Correspondence: Huai-Dong Chen, Department of Cardiac Surgery, Sir Run Run Shaw Hospital, School of Medicine, Zhejiang University, Hangzhou, China; (e-mail:3317025@zju.edu.cn). adhesion, thus lowering the risk of needing to redo a cardiac procedure in the future.

\section{INTRODUCTION}

Median sternotomy is the most common surgical approach for mitral valve replacement. However, this type of surgery leaves larger wounds, resulting in prolonged recovery time, and can cause serious complications such as infection, wound dehiscence, and even death, affecting treatment efficacy to a certain extent. With the development of minimally invasive surgical techniques, cardiovascular surgery is gradually becoming minimally invasive [Woo 2006]. In 1997, Chitwood et al [Chitwood 1997] reported they performed thoracoscopically assisted mitral valve surgery through a $4-\mathrm{cm}$ small incision on the right side of the chest wall and achieved satisfactory clinical outcomes. Owing to its various merits such as less trauma, shorter postoperative recovery time, reduced operation costs, and improved cosmetic results [Chitwood 1997], thoracoscopic cardiac surgery rapidly has improved from being a thoracoscopically assisted treatment to being a totally thoracoscopic cardiac surgery (TTCS). However, some surgeons remain concerned that restricted exposure, limited operating space, and longer instruments may lead to inferior results in mitral valve surgery [Baldwin 1998]. The aim of our study was to review our previous collective results and to examine the efficacy, safety, and early outcomes of totally thoracoscopic mitral valve replacement.

\section{MATERIALS AND METHODS}

\section{Clinical Data}

This study included 94 patients, who underwent mitral valve replacement surgery (MVR) between January 2016 and December 2017. In our study, patient inclusion criteria were as follows: (1) patients without thoracic deformity with a BMI $\leq$ $30 \mathrm{~kg} / \mathrm{m}^{2}$; (2) patients with pure mitral valve disease (or coexisting with tricuspid regurgitation); (3) patients with $\mathrm{PaO}_{2} \geq 80 \%$ as confirmed by the arterial blood gas analysis at admission; and (4) patients with concern over the incision size.

\section{Contraindications were as follows}

(1) a history of right chest wound or surgery; (2) history of lower extremity arterial disease; (3) history of coronary heart 
disease; (4) severe mitral annulus calcification; (5) coexistence of left atrial thrombus; and (6) coexistence of other cardiac malformations. All patients who accorded with the inclusion criteria were divided into the thoracoscopic and traditional groups, according to patient selection. The Ethics Committee of Clinical Trials and Biomedical (Sir Run Run Shaw Hospital, School of Medicine, Zhejiang University) approved this study, and all patients signed informed consent forms.

The thoracoscopic group consisted of 43 patients, including 12 men and 31 women, age 23 years to 66 years (mean \pm $\mathrm{SD}, 46.23 \pm 8.72$ years). The mean body mass index (BMI) of the patients was $22.80 \pm 2.93 \mathrm{~kg} / \mathrm{m}^{2}$. Of the patients, $23.3 \%$ (10 out of 43 ) had a BMI $\geq 25 \mathrm{~kg} / \mathrm{m}^{2}$. All patients definitively were diagnosed based on history, cardiovascular signs, electrocardiographic (ECG) findings, echocardiographic results, and other test results. The diagnoses included 30 cases of isolated mitral stenosis, three cases of mitral regurgitation, eight cases of tricuspid regurgitation, and 10 cases of coexisting mitral stenosis and regurgitation. ECG showed atrial fibrillation in 22 cases. Echocardiogram showed a left ventricular end-diastolic dimension (LVEDD) of $47.07 \pm 3.22 \mathrm{~mm}$ and an ejection fraction (EF) of $58.40 \% \pm 3.51 \%$. In particular, 17 patients had class I/II preoperative cardiac function and 26 patients had class III/IV, according to the New York Heart Association (NYHA) classification system.

The traditional group consisted of 51 patients, including 15 men and 36 women, age 27 years to 70 years (mean \pm SD, $49.88 \pm 10.31$ years). Their mean BMI was $22.78 \pm 3.05 \mathrm{~kg} /$ $\mathrm{m}^{2}$. Of the patients, $29.4 \%$ (13 out of 51 ) had a BMI $\geq 25 \mathrm{~kg} /$ $\mathrm{m}^{2}$. The diagnoses included 35 cases of isolated mitral stenosis, four cases of mitral regurgitation, 12 cases of coexisting mitral stenosis and regurgitation, and 18 cases of tricuspid regurgitation. ECG showed atrial fibrillation in 24 cases. Echocardiogram observations revealed a LVEDD of $48.71 \pm$ $5.53 \mathrm{~mm}$ and an $\mathrm{EF}$ of $59.16 \% \pm 4.68 \%$. In particular, 21 patients had NYHA class I/II preoperative cardiac function, and 30 patients had class III/IV.

The two groups did not show statistically significant differences with respect to sex, age, BMI, LVEDD, EF, and NYHA. The patients' preoperative data are shown in Table 1.

Surgical Procedures: For the thoracoscopic group, each patient underwent a single-lumen endotracheal intubation under general anesthesia. The surgery was performed under high-frequency ventilation with low tidal volume. The patient was placed in the supine position, with the right chest elevated $20^{\circ}$ to $30^{\circ}$ and the right upper arm fixed to a head frame. A small vertical incision was made under the right groin where the femoral artery pulse was palpable to isolate the femoral artery and vein. An arterial catheter $(15 \mathrm{~F}-20 \mathrm{~F})$ and a venous catheter (21F-25F) (Medtronic, Minneapolis, Minn) were inserted respectively after injection with heparin $(3-\mathrm{mg} / \mathrm{kg})$.

Three small incisions were made in the right side of the chest wall. The first one was for the insertion of the thoracoscope and was located near the right midaxillary line in the fourth intercostal space (ICS), $1 \mathrm{~cm}-1.5 \mathrm{~cm}$ in length. The second one was for the right-hand instruments, such as needle forceps, shears and aspirator, and was located at the right parasternal fourth ICS, deviating slightly outward
Table 1. Preoperative Patient Characteristics

\begin{tabular}{lccc}
\hline & $\begin{array}{c}\text { Thoracoscopic } \\
\text { Group (N=43) }\end{array}$ & $\begin{array}{c}\text { Traditional } \\
\text { Group (N=51) }\end{array}$ & $P^{*}$ \\
\hline Age (years) & $46.23 \pm 8.72$ & $49.88 \pm 10.31$ & .070 \\
Sex, Male (\%) & $27.9 \%(12 / 43)$ & $29.4 \%(15 / 51)$ & .872 \\
BMI (Kg/m², mean) & $22.80 \pm 2.93$ & $22.78 \pm 3.05$ & .975 \\
$\geq 25$ (Kg/m², \%) & $23.3 \%(10 / 43)$ & $25.5 \%(13 / 51)$ & .802 \\
LVEDD (mm) & $47.07 \pm 3.22$ & $48.71 \pm 5.53$ & .078 \\
Ejection fraction (EF\%)(mean) & $58.40 \pm 3.51$ & $59.16 \pm 4.68$ & .371 \\
NYHA class & & & .872 \\
I II & $(17 / 43)$ & $(21 / 51)$ & \\
III IV & $(26 / 43)$ & $(30 / 51)$ & \\
Rhythm & & & \\
Sinus rhythm (\%) & $48.8 \%(21 / 43)$ & $52.9 \%(27 / 51)$ & .692 \\
Atrial fibrillation (\%) & $51.2 \%$ & $47.1 \%$ & \\
Echocardiographic findings & & & \\
Mitral regurgitation (MR,\%) & $6.98 \%(3 / 43)$ & $7.84 \%(4 / 51)$ & .986 \\
Mitral stenosis (MS,\%) & $69.77 \%(30 / 43)$ & $68.63 \%(35 / 51)$ & \\
MS+MR (\%) & $23.26 \%(10 / 43)$ & $23.53 \%(12 / 51)$ & \\
With diseases & & & .218 \\
Tricuspid insufficiency (\%) & $18.6 \%(8 / 43)$ & $39.22 \%(18 / 51)$ & .072 \\
Diabetes (\%) & $4.65 \%(2 / 43)$ & $11.76 \%(6 / 51)$ & .134 \\
Hypertension (\%) & $16.28 \%(7 / 43)$ & $29.41 \%(15 / 51)$ &. \\
\hline & & & \\
\hline
\end{tabular}

Data presented as mean \pm SD or number $(\%)^{*}$ Thoracoscopic group versus traditional group. BMI= body mass index; LVEDD= left ventricular enddiastolic dimension; NYHA = New York Heart Association

approximately $1 \mathrm{~cm}$ parasternal to avoid the internal mammary artery, approximately $3 \mathrm{~cm}-4 \mathrm{~cm}$ in length. The third one was for the insertion of an aortic cross clamp and lefthand instruments, and was located at the third ICS on the right anterior axillary line, $1 \mathrm{~cm}-1.5 \mathrm{~cm}$ in length.

After the thoracoscope properly was placed, an incision was made in the pericardium, above the right phrenic nerve with the thoracoscope guidance. A ligature was placed in the right atrial appendage, with the string pulled out from the first hole to pull the right atrial downward to expose the ascending aortic root and superior vena cava (SVC). A purse-string suture was sewn at the root of the ascending aorta to insert an antegrade cardioplegia needle. A transthoracic aortic crossclamp was used to occlude the ascending aorta via the third hole. The antegrade perfusion with cold cardioplegia solution was performed. After the cardiopulmonary bypass (CBP) began, the SVC and inferior vena cava (IVC) were dissected with an umbilical tape.

After cardiac arrest, an incision was made parallel to the atrioventricular groove to open the left atrium. A special atrial retractor was placed inside and pulled to expose the mitral valve. The mitral leaflets completely were removed, and the 
Table 2. Surgical Procedures and Operative Times

\begin{tabular}{|c|c|c|c|}
\hline Variables & $\begin{array}{c}\text { Thoracoscopic } \\
\text { Group } \\
(\mathrm{N}=43)\end{array}$ & $\begin{array}{l}\text { Traditional } \\
\text { Group } \\
(\mathrm{N}=51)\end{array}$ & $P^{*}$ \\
\hline \multicolumn{4}{|l|}{ Valve replacement type } \\
\hline Mechanical valves & 42 & 47 & .235 \\
\hline Biological valves & & 1 & 4 \\
\hline Tricuspid valvuloplasty & 8 & 18 & .072 \\
\hline \multicolumn{4}{|l|}{ Operative times ( $\mathrm{min}$ ) } \\
\hline Cross-clamp time & $62.30 \pm 8.17$ & $44.90 \pm 12.00$ & .000 \\
\hline Cardiopulmonary bypass time & $92.33 \pm 12.03$ & $74.22 \pm 14.72$ & .000 \\
\hline Operative time & $184.26 \pm 32.49$ & $181.47 \pm 23.31$ & .631 \\
\hline Mechanical ventilation $(h)$ & $10.14 \pm 2.21$ & $11.35 \pm 2.58$ & .016 \\
\hline Intensive care unit stay times $(\mathrm{h})$ & $21.40 \pm 3.15$ & $29.12 \pm 6.59$ & .000 \\
\hline Postoperative drainage $(\mathrm{ml})$ & $325.72 \pm 97.11$ & $396.57 \pm 121.5$ & .000 \\
\hline Postoperative hospital times (days) & $8.70 \pm 2.52$ & $10.04 \pm 3.11$ & .023 \\
\hline
\end{tabular}

Data are presented as mean \pm SD or number (\%)

* Thoracoscopic group versus traditional group

posterior mitral leaflets were preserved whenever possible. The double-armed, mattress sutures were placed counterclockwise from the 11:00 position. Every stitch directly was placed in the prosthesis-sewing ring outside of the chest. The prosthesis was put into the chest, and the knots were tired with the right middle finger of the operator. For the eight patients with tricuspid regurgitation, the tricuspid valvuloplasty with MC3 ring (Edwards) also was performed. Before the incision in the atrioventricular groove was stitched, air was forced out through the antegrade cardioplegia needles in the ascending aorta. Then, the ascending aorta was released. The incision in the right atrium was then closed with a 4-0 prolene suture (Ethicon). The patients were weaned from $\mathrm{CPB}$, and the $\mathrm{CPB}$ was terminated. A drainage tube was inserted into the right thorax cava through the first port. Transesophageal echocardiography confirmed that the mitral prosthetic valve successfully worked.

For the traditional group, surgery was performed through a median sternotomy. The tricuspid valvuloplasty with MC3 ring (Edwards) also was performed for 18 cases with tricuspid regurgitation.

The same surgical team performed all the procedures.

Postoperative care and follow-up: The patients were sent to the ICU after surgery. The tracheal tube was removed after the patient was fully awake and able to independently breathe well; the arterial blood gas values were restored to within the reference ranges. Most patients were transferred to the general ward on the day of surgery or one day after the operation and began to receive an anticoagulation therapy. In general, when the amount of drainage from the chest tube was $<100$ $\mathrm{mL} / \mathrm{d}$ and the chest radiograph showed that lung recruitment was progressing well, the drainage tube was removed.
Table 3. Results of Clinical and Echocardiographic Follow-Up

\begin{tabular}{lccc}
\hline Variables & $\begin{array}{c}\text { Thoracoscopic } \\
\text { Group } \\
(\mathrm{N}=43)\end{array}$ & $\begin{array}{c}\text { Traditional } \\
\text { Group } \\
(\mathrm{N}=51)\end{array}$ & $P^{*}$ \\
\hline Hospital mortality & 0 & 0 & \\
Postoperative complications & $3(6.98 \%)$ & $4(7.84 \%)$ & .873 \\
Pneumothorax & 2 & 0 & \\
Wound infection & 0 & 2 & \\
Pneumonia & 0 & 1 & \\
Second surgical & 0 & 1 & \\
Hemostasis & 1 & & \\
Arrhythmia (bradycardia): & & & \\
Pacer & $12.09 \pm 1.76$ & $11.25 \pm 2.12$ & .039 \\
$\quad$ Mean follow-up (months) & & & \\
Echocardiographic & & & \\
Follow-up & & & \\
LVEDD (mm) & & & \\
Ejection fraction (\%) & $60.86 \pm 3.78$ & $61.55 \pm 4.64$ & .430 \\
NYHA class & & & .958 \\
I II & $23(53.49 \%)$ & $27(52.94 \%)$ & \\
III IV & 20 & 24 & \\
Mild tricuspid regurgitation & $26.53 \pm 2.39$ & $46.04 \pm 4.53$ & .501 \\
\hline
\end{tabular}

Data are presented as mean \pm SD or number (\%)

* Thoracoscopic group versus traditional group

Echocardiography and electrocardiography were performed during follow-up.

Statistical analysis: All analyses were performed using SPSS 20.0 statistical software (IBM SPSS Inc. Chicago, IL), and continuous data were presented as mean \pm standard deviation whereas non-normally-distributed data were presented as median and interquartile range and frequencies as proportions. Between-group differences were compared using the $t$ test, and numerical data were compared using the $\chi^{2}$ test, with a $P<.05$ considered as significantly different.

\section{RESULTS}

The thoracoscopic group consisted of 43 patients, of whom 42 received a mechanical valve (St Jude Medical, St Paul, Minn) and the remaining one received a bioprosthetic valve (Carpentier-Edwards; Edwards Lifesciences, Irvine, Calif). The traditional group consisted of 51 patients, of whom 47 cases had a mechanical valve replacement (St Jude Medical, St Paul, Minn) and four had a bio-prosthetic valve replacement (CarpentierEdwards; Edwards Lifesciences). The patients in the thoracoscopic group had longer aortic clamping and CPB times than those in the traditional group $(62.30 \pm 8.17$ minutes versus 44.90 
\pm 12.00 minutes, $P<.001$ and $92.33 \pm 12.03$ minutes versus $74.22 \pm 14.72$ minutes, $P<.001$, respectively). The difference in operative time between the two groups was not statistically significant $(184.26 \pm 32.49$ minutes versus $181.47 \pm 23.31$ minutes, respectively, $P=.631$ ) (Table 2 ). In addition, the postoperative mechanical ventilation, ICU stay, and postoperative hospital stay times and postoperative drainage were $10.14 \pm 2.21$ hours and $11.35 \pm 2.58$ hours $(P=.016), 21.40 \pm 3.15$ hours and $29.12 \pm$ 6.59 hours $(P<.001), 8.70 \pm 2.52$ days and $10.04 \pm 3.11$ days $(P=$ $.023)$, and $325.71 \pm 97.11 \mathrm{~mL}$ and $396.57 \pm 121.50 \mathrm{~mL}(P<.001)$ for the thoracoscopic group and traditional group, respectively.

The postoperative complication incidence rates in the two groups were not statistically significant. The thoracoscopic group had three cases $(6.98 \%)$ of postoperative complications, including two cases of right-side pulmonary atelectasis and one case of temporary pacemaker installation due to sinus bradycardia. An echocardiographic examination upon hospital discharge showed that the prosthesis was in a proper position and worked well without any paravalvular leakage. Twenty patients had a NYHA class I/II cardiac function, and 23 patients had a NYHA class III/IV cardiac function. Four patients $(7.84 \%)$ in the traditional group had complications, of whom one had nosocomial pulmonary infection, two had a wound infection that resulted in longer healing time, and one had postoperative bleeding that required a second surgical hemostasis. For the traditional group, an echocardiographic examination upon hospital discharge revealed that the prosthesis was functioning well, and had no paravalvular leakage. Cardiac function was classified as NYHA class I/II in 26 patients and class III/IV in 25 patients. The details are presented in Table 3.

The mean follow-up durations for the two groups were $12.09 \pm 1.76$ months and $11.25 \pm 2.12$ months $(P=.039)$, respectively. No patients in either group had complications during the follow-up period. Clinical follow-up was $100 \%$ complete for the thoracoscopic group and 96.08\% (49 out of 51) complete for the traditional group. There were two $(3.92 \%)$ patients from the traditional group whose echocardiographic follow-up after hospital discharge was not performed (the address and phone number registered with the hospital had changed). For the patients who were lost to follow-up, the latest observed data were used for the data analysis.

Echocardiographic results obtained from postoperative follow-up showed no statistically significant differences in LVEDD and EF between the two groups of patients $(46.53$ $\pm 2.39 \mathrm{~mm}$ versus $46.04 \pm 4.53 \mathrm{~mm}, P=.501 ; 60.86 \pm 3.78 \%$ versus $61.55 \pm 4.64 \%, P=.430)$. NYHA functional class improved significantly in both groups, demonstrating no statistical difference between the groups $(P=.958)$. Mild tricuspid regurgitation was found in two patients of the thoracscopic group and in four patients of the traditional group. The details are presented in Table 3.

\section{DISCUSSION}

The most common surgical approach to treat mitral valve disease is to completely split the sternum and directly access the heart for operation. While this approach provides excellent exposure for heart operations, the resulting trauma takes months to fully heal, leading to prolonged recovery and significant limitation on physical activities. It also can cause serious complications, including infection, wound dehiscence, and even death. In our study, the patients in the traditional group had a significantly higher amount of postoperative drainage than the patients in the thoracoscopic group. Furthermore, compared with the thoracoscopic group, the traditional group had two cases of local infection around the incisions that resulted in prolonged healing time and one case of bleeding that required a second surgical hemostasis.

Until now, although many reports on totally thoracoscopic cardiac surgery have been published, no unified standards have been established to identify good candidates for surgery. The identification generally depends on the cardiac surgeon's clinical experience and proficiency level for minimally invasive valve surgeries. For the patients with obesity, some researchers [Suri 2012] previously suggested that obesity should be treated as a surgical contraindication, but no clear criteria were presented. For our study, the BMI standard we chose was $\leq 30 \mathrm{~kg} / \mathrm{m}^{2}$. Twelve patients had a BMI of $25-30 \mathrm{~kg} / \mathrm{m}^{2}$ and successful operations. However, it is worth noting that because of the thicker subcutaneous fat layer in obese patients, operators should be careful to avoid fat particles around the incision falling into the heart chambers, which may cause complications such as infarction. Before closing up the three small ports after the completion of operation, the operator should carefully check for bleeding or any small intercostal artery injury to avoid situations, including excessive postoperative drainage or a second operation to stop the bleeding.

Perfect exposure is a core issue in various minimally invasive techniques used in cardiac surgeries. Regardless of whether it is in small-incision, robot-assisted, or total thoracoscopic cardiac surgery, neither good exposure nor patient safety can be compromised. Only procedures that allow for visual clarity that are safe, effective, and can yield comparable or superior results to that of conventional surgical approaches are worthy of wide application. In our study, the video-assisted thoracoscopic technology can provide surgeons a clear magnified two-dimensional surgical field $(10 \mathrm{~mm})$. Also in our study, all patients in the thoracoscopic group had a successful surgery, and none required additional incision during operation. The differences in postoperative complications between the two groups were not statistically significant (three cases versus four cases). During the follow-up period, no patient in either group died or required a second surgery. Echocardiographic examination showed no statistically significant difference in LVEDD and EF (\%) between the two groups.

Under the premise of sufficient exposure, we believe the following points are also important for the success of totally thoracoscopic mitral valve replacement surgery: (1) When exposing the ascending aortic root, the operator could pull the top right atrial and then pull the string out of the second port to get a clarifying exposure. In addition, when a pursestring suture is being sewn in the ascending aorta, a long Allis clamp can be used to pull up the aortic outer membrane for 
the convenience of operating. (2) We chose to sew the mitral valve counterclockwise from the 11:00 position, and the operation of sewing the remnant valve with the mitral mechanical valve was performed outside of the chest to avoid suture tangling. (3) When tying knots, we chose to use the right middle finger to push down the knot. This is because when using a specialized knot-tying device, the strength applied to knot tying cannot be accurately controlled. However, for a patient with a deeper chest, a device must be used because the right middle finger may not be as effective.

Compared with the traditional group, the patients in the thoracoscopic group had a shorter postoperative ICU monitoring period, mechanical ventilation duration, and hospital stay. Patients in the thoracoscopic group had the shortest hospital stay (four days); the traditional approach group had a longer stay (6 days).

The development and application of new technologies inevitably will require a certain learning curve and skill development cycle. Previous studies have reported that because minimally invasive cardiac surgeries are performed in a confined space with higher difficulty level, the CPB duration, aortic cross-clamp times, and operative times are all longer compared with those in the traditional surgery group [Baldwin 1998; Lucà 2013; Brown 2009]. Our study derived similar results in that the thoracoscopic group had longer $\mathrm{CPB}$ duration and aortic cross-clamp times than the traditional group. However, interestingly, in our study, the operative durations in the two groups were not significantly different $(184.26 \pm 32.49$ minutes versus $181.47 \pm 23.31$ minutes, respectively; $P=.631$ ). We deduced that the possible reasons are as follows: First, because of the smaller ports and less bleeding in the patients of the thoracoscopic group, the times spent on the hemostasis after intracardiac operation and closure of the small ports were shorter, resulting in a shorter overall operative time than with the traditional group. Second, our surgical team completed more than 100 totally thoracoscopic cardiac surgeries and has accumulated a rich experience [Cheng 2013], thus the surgical procedures were accelerated. The results reported by Gao et al stated that with surgeons' shortened learning curve and increased surgical experience, the cross-clamp and bypass times will be apparently shortened as well [Gao 2012]. Therefore, we believe that with surgeons becoming more skilled with thoracoscopic surgeries and the learning curve getting shorter, CPB duration and cross-clamp times also will shorten, which ultimately will reach a similar level as in conventional sternotomy.

\section{LIMITATIONS}

Limitations of our study are as follows: First, this study was a single-center case-control study that consisted only of a limited number of patients. Follow-up period was relatively short, thus, the information regarding the patients' longterm recovery and complications was not clear. No unified standard of patient inclusion criteria was established for the thoracoscopic group, which needs to be further explored to clarify the safety and efficacy of this technology in patients at various stages of the disease.

\section{CONCLUSION}

Despite disadvantages including long cross-clamp and cardiopulmonary bypass times, the totally thoracoscopic mitral valve replacement showed multiple advantages. These advantages include less trauma, shorter hospital stay, postoperative intact thoracic cavity, and good cosmetic appearance. Moreover, we believe video-assisted thoracoscopic cardiac surgery also has the following benefits: (1) It can provide a clear magnified visual field of the lesion area for the surgeons, and (2) it can avoid retrosternal adhesion compared with the traditional median thoracotomy, thus lowering the risk of reperforming a cardiac procedure.

We believe that the totally thoracoscopic mitral valve replacement is feasible and safe. And, with the increasing technological development and surgical procedural proficiency, video-assisted thoracoscopic cardiac surgery will become fully developed and eventually replace the traditional median thoracotomy and be the preferred standard surgical treatment.

\section{ACKNOWLEDGMENTS}

This work was supported by a grant from Medical Health Science and Technology Project of Zhejiang Provincial Health Commission(No.2016ZDA013)".

\section{REFERENCES}

Baldwin JC. 1998. Editorial (Con) Re Minimally invasive port-access mitral valve surgery. J Thorac Cardiovasc Surg, 115: 563-4.

Brown ML, McKellar SH, Sundt TM, Schaff HV. 2009. Ministernotomy versus conventional sternotomy for aortic valve replacement: A systematic review and meta-analysis. J Thorac Cardiovasc Surg, 137:670.

Cheng Y, Chen H, Mohl W, Liu X, Si Z. 2013. Totally endoscopic congenital heart surgery compared with the traditional heart operation in children [J]. Wien Klin Wochenschr, 125(21-22): 704-708.

Chitwood WR Jr, Elbeery JR, Chapman WH, Moran JM, Lust RL, Wooden WA, et al. 1997. Video-assisted minimally invasive mitral valve surgery: the "micro-mitral" operation[J]. J Thorac Cardiovasc Surg, 113(2): 413-414.

Gao C, Yang M, Xiao C, Wang G, Wu Y, Wang J, et al. 2012. Robotically assisted mitral valve replacement [J]. J Thorac Cardiovasc Surg, 143(4): S64-S67.

Lucà F, Van G L, Rao C M, et al. 2013. Minimally Invasive Mitral Valve Surgery: A Systematic Review[J]. Minimally Invasive Surgery (2013-327), 2013(2):179569.

Suri R M, Thalji N M. 2012. Minimally invasive heart valve surgery: How and Why in 2012[J]. Current Cardiology Reports, 14(2): 171-179.

Woo Y J. 2006. Robotic cardiac surgery. Int J Med Robot, 2(3): 225-232. 